\title{
Resilience of Interconnected Infrastructures and Systems: the RESIIST project
}

\author{
Daouda KAMISSOKO ${ }^{1}$, Blazho NASTOV ${ }^{2}$, Vincent CHAPURLAT ${ }^{3}$, Hélène DOLIDON ${ }^{4}$, \\ Aurelia BONY-DANDRIEUX ${ }^{3}$,Bruno BARROCA ${ }^{5}$, Mickael MARECHAL $^{6}$, Jerome TIXIER ${ }^{3}$, \\ Matthieu ALLON ${ }^{2}$, Frederick BENABEN ${ }^{1}$, Nicolas DACLIN ${ }^{3}$, Alexis MULLER ${ }^{2}$, Nicolas \\ SALATGE $^{1}$, Valerie NOVEMBER ${ }^{5}$ \\ ${ }^{1}$ IMT Albi Mines, University of Toulouse, France \\ \{douda.kamissoko, frederock.benabeb, nicolas.salatge\}@mines-albi.fr \\ 2 Axellience, Lille, France \\ \{blazho.nastov, matthieu.allon, alexis.muller\}@axellience.com \\ 3 IMT Mines Alès, Alès, France \\ \{vincent.chapurlat, aurelia.bony-dandrieux, jerome.tixier, nicolas.daclin\}@mines-ales.fr \\ ${ }_{4}$ CEREMA, Nantes, France \\ helene.dolidon@cerema.fr \\ ${ }_{5}$ Université Paris-Est, Marne-la-Vallee, France \\ \{bruno.barroca, valerie.november\}@u-pem.fr \\ ${ }_{6} \mathrm{SNCF}$, Toulouse, France \\ mickael.marechal@sncf.fr
}

\begin{abstract}
This paper introduces a methodology for resilience assessment of critical infrastructures based on massive data. The methodology is developed for the needs of the RESIIST research project. We start from the observation that the security of large cities has become a major issue. To ensure the proper functioning of critical infrastructures, it is essential to make the right decisions at the right time. To do this, managers are informed in their decision-making processes by several indicators such as resilience. As insecurity becomes more and more threatening with technological, natural and terrorist risks, it is essential to have an indicator of resilience of the infrastructures guaranteeing security. We therefore propose an innovative method of assessing resilience. It is innovative in that it combines both the genericity (it applies to all types of infrastructure), it takes into account several dimensions (economic, technical, social, human, regulatory etc.), it integrates massive data (from cameras, sensors, GIS, and social networks), it allows decision-making in an immersive environment in virtual reality.
\end{abstract}

Keywords: Resilience, Critical Infrastructure, Decision Making, Big Data, Simulation, Virtual Reality, Security, System.

\section{Introduction}

This paper presents the proposal of the RESIIST project (https://research-gi.minesalbi.fr/display/resiist/RESIIST+Home). It addresses the issues of resilience assessment of critical infrastructures guaranteeing the security of territories. The term "critical infrastructure" is here-defines as "an infrastructure whose well-functioning is an issue for the stability and the functioning of a territory". Examples of critical infrastructure include power generation and distribution systems, water, gas, health services, banking, state (schools, laboratories, town halls, courts), or companies producing goods and services (industries, agribusiness, public services). The term 
"resilience" is here-defined as "the ability of an infrastructure to interact with other infrastructures and its environment in order to fulfill its missions and provide the expected services while facing different risks". Risk is used in the sense of a feared event (natural disaster, terrorist attack, internal dysfunction).

The idea of this paper is based on the fact that nowadays it is impossible to imagine a society without considering the preponderant role of critical infrastructures and systems that surround us. In an increasingly liberalized global economy, society needs are changing, but are still based on the assumption that critical infrastructures are functioning [1], [2]. Critical infrastructures show an increased vulnerability. From a resilience perspective, critical infrastructure observation makes the following finding. The environment and needs are changing: Critical infrastructures are evolving in environments where continuous delivery of goods and services is required. The demands that regulate their operations constantly evolve and affect the critical infrastructures in the execution of their societal missions. For example, in France, electricity consumption has increased by $350 \%$ in 40 years. Everything is connected to everything, and everything interacts with everything: Critical infrastructures, products and even entire cities become interconnected. They regularly provide some information on their statements and receive other information to fulfill their missions. Connectivity and interdependencies make infrastructures complex and lead to cascading failures and unpredictable behavior that can reduce the resilience [4]. Risk situations are growing: Whether in a critical infrastructure, a country, a city or a community, disruptive events occur daily. Instability has become the norm to integrate [1]. The assessment made by the company F-Secure in terms of computer security finally highlights 99 countries victims of computer attacks for the sole month of May 2017. In addition, the loss experience is diversified: The risks do not spare any structure. The specter of consequences is becoming wider: human lives, territory, economy, or branding are the targets with increasingly dramatic repercussions.

From this observation, several scientific and technological issues arise: The first is "how to identify critical infrastructures and their limits?". Indeed, with connectivity and interdependencies, the boundaries of an infrastructure are not obvious to identify. There is a real need for all stakeholders to define the reasonable limits of their infrastructure. The second is "how to understand the critical infrastructure at any time and in confidence, by crossing at best several data existing but not necessarily used?". This question considers the fact that infrastructures - interacting with each other, behaviors and properties that are difficult to deduce can emerge. There is therefore a need for data and models to improve the understanding of critical infrastructures. The third issue is "how to decide and act effectively?". Cost has long been the key element in critical infrastructure decision-making. This reality is challenged by the need to consider other dimensions considered today as crucial for a 
better control of resilience (ecological, human, social, political). In addition, it is essential to consider several often-conflicting points of view (users, managers, communities, associations, lobbies). The fourth and last issue is "how to justify decisions?". Since decision-making is not an end, to be accepted, every decision must be justified and rationalized. Rationalization gives a scientific argument to the decision and increases its acceptability.

Based on these observations and the resulting issues, our idea is to use big data to continuously evaluate the resilience of critical infrastructures. Data from various sources is interpreted to provide relevant indicators reflecting several dimensions. By using these indicators, models of infrastructure and resilience are developed. The intelligent visualization of these models makes it possible to take and justify decisions. Thus, the purpose of this paper is to propose a generic methodology for continuous resilience assessment that consider (i) model of a critical infrastructure (ii) continuous flows of big data (iii) the identification of decisions and their implementation (iv) the integration of several dimensions and several points of view. The big data sources used are from various natures (Cameras, Social Networks, Sensors, OpenData, GIS). From a societal point of view, with our proposal, it becomes possible to continuously monitor the critical infrastructures and territories on which they are called to function in any situation.

To fulfil this objective, several questions form the problematic of this paper: (1) Is it possible to continuously measure the resilience of an infrastructure, an organization, a territory with a generic approach? (2) How to prioritize infrastructures and determine the most critical one according to the context? (3) What differentiates one infrastructure from another for a given context and what is needed to put forward to characterize a critical infrastructure considering multiple points of view? (4) What are the risks likely to affect the proper functioning? (5) What are the actions to be defined preventively and correctively? (6) What could be the consequences of a decision and how can it be justified considering the analysis of the potential consequences?

To answer these questions, we propose a process that involves three steps: the interpretation of data into information, the exploitation of information into knowledge, the decision making for obtaining actions to do. The data streams are monitored continuously to obtain big data present in the data layer. The use of big data for the evaluation of resilience is the first originality of this approach. During the interpretation phase, the data provides indicators for infrastructure and risk. The indicators represent several dimensions including at least the technical, economic, environmental, regulatory, social and human dimensions. All these dimensions constitute the second originality. The context is enriched by a study of risks, impacts (resulting from the occurrence of the risk) and corrective and / or preventive treatment strategies. The implementation of these strategies has a systematic impact on the indicators 
and greatly reduces the consequences of the risk. This in-depth risk engineering is the third originality. The indicators are used in knowledge to obtain a model of resilience and a representation / simulation model of the infrastructure. The latter is represented by its digital twin and its resilience is evaluated considering the lack of knowledge, inaccuracies or uncertainties. It is thus possible to steer the infrastructure through its overall resilience. The use of the digital twin of the infrastructure is the fourth originality. Continuous evaluation of the infrastructure resilience in real time is the fifth originality. The decision-making process results in recommendations and a rational justification for them. Considering the points of view of all stakeholders is the sixth originality. Smart dashboards combined with virtual support the representation, steering and support for the implementation of the decision is the seventh and last originality of this paper.

For the relevancy of our proposal, we took care to make a state of the art on all issues related to the resilience of critical infrastructure. Then we built a case study to test the feasibility of our proposals. The next section describes the literature review.

\section{Literature review}

This section presents the current state of the are concerning the needs mentioned above. We present three issues in the management of infrastructures in relation to the needs: the problematic of evaluation of the resilience, the problematic of modeling, and the problematic of decision.

\subsection{Resilience Assessment}

Resilience is a concept of growing interest to the scientific community. In Science Direct referencing of scientific articles, from 63 articles containing the word "resilience" in the title in 2007, we moved to 631 articles in 2017, an evolution of $1000 \%$ in just 10 years. The concept of resilience and the definitions that refer to it depend on the domain [2]. Whatever the field considered, the notion of resilience generally implies the presence of a risk and refers to the loss of a performance indicator. In the literature, there are different ways of dealing with infrastructure resilience issues: classical, qualitative, quantitative, data, expertise, and feedback. The traditional approach is to apply a traditional risk analysis approach to identify and address a risk for a specific infrastructure [3] [4]. Previous findings in terms of interdependence and connectivity make traditional approaches insufficient to adequately analyze the resilience of an infrastructure. The qualitative assessment family contains conceptual models and approaches based on the estimation of a semiquantitative index. It is made particularly difficult by considering several dimensions or evaluation criteria and the possible inconsistency between these criteria. The family of quantitative methods can be decomposed into models of simulation, 
optimization, fuzzy logic, stochastic and deterministic. The reader is invited to see [5] for more information on this category. Evaluating resilience from data is done through data streams from various sources [6]. An evaluation by the transmitted data generates a complexity and issues related to the speed of emission, the quantity of the data emitted, and the diversity of their nature. In evaluation by expertise, the views of one or more experts are modeled and aggregated to obtain a global indicator [7]. In this evaluation category, the capture of antagonistic points of view and their conciliation constitute the main issue. Feedback assessment is mainly practiced in social resilience [8]. It consists of doing similarity analyzes in a situation from past cases. The identification of cases and similarities is the issue of this category.

\subsection{Resilience Assessment}

We mean by modeling the activity leading to the representation of the original infrastructure [9]. The problem of modeling is a crucial problem when it comes to critical infrastructure systems [10]. The systemic approach and several methods such as those proposed in [11], [12] or [13] are then recommended to be able to master, and to facilitate the understanding of the factors inducing this apparent or real complexity. In the field of engineering sciences, the System Engineering approach (ISO 15288) promotes standardized processes for the definition of specifications, design, analysis, verification and validation. and the use of models [14], [15], [16], [17]. In this view, a system is usually modeled using a multi-view approach based at least on five points of view: (1) system, (2) need (3) functional and logical, (4) physical / organic and (5) behavioral. The system point of view represents the main features of the system as it currently exists (we are talking about AS-IS model) or as it should be (TO-BE model and sometimes TO-IMPLEMENT model) and its boundaries with the environment. The point of view needs defined the specifications and constraints of different stakeholders. The functional and logical point of view defines different functional architecture solutions of the system. The organic then physical point of view [18] allows the representation of the variants of the concrete architecture of the system. The definition or the choice of the DMSL (Design Specific Modelling Languages), favoring a systemic approach, and the construction of a numerical model is thus the first scientific issue of the modeling. The digital model must also be enriched with data, information and external knowledge, not necessarily easy to model. They must therefore be integrated and enrich the digital model to form what is then called the digital twin to meet the need for simulation and continuous evaluation of the resilience [19] of all or part of the critical infrastructure [20], [21], [22]. The mastery of data constraints used for the digital twin (temporalities, the risk of obsolescence) is the first issue of modeling. Transforming data models into indicators to build the infrastructure model is the second issue of the modeling. 


\subsection{Decision Support}

Literature review in decision-making in the field of critical infrastructures reveals three types of problems: identification of criteria, procedure of aggregation of criteria, visualization of decisions and implementation of decisions. The identification of the decision criteria is a major stake in the management of the critical infrastructures [23], [24]. The criteria identification methodology proposed by [23] contains two essential steps: (1) determination of objectives and scope and (2) consultation, analysis of acceptability, reasonableness and realism. To our knowledge, there is no set of criteria or methodology applicable to each situation. This is explained by the principle of limited rationality in which (i) the decision-making environment is too complex to be comprehensively apprehended, (ii) the knowledge of the consequences of a decision is always partial, (iii) the rationality of an individual is limited due to lack of time and cognitive ability [25]. However, several authors agree that the criteria must consider several dimensions and check the axioms of completeness, cohesion and non-redundancy defined in [26]. The problem of aggregation lies in the search for a compromise between several criteria and / or the aggregation of the points of view of multiple decision-makers. There is a legion of aggregation procedures in the literature and so many ways of categorizing them. The most widely used methods are: Multi-Attribute Utility Theory, AHP (Analytic Hierarchy Process), Fuzzy Set Theory, Case-based Reasoning, Data Envelopment Analysis, Goal Programming, ELECTRE, PROMETHEE, TOPSIS and the weighted sum [27]. (Triantaphyllou, 2000) recommends a classification according to the type of data (Deterministic, Stochastic, Fuzzy) and the number of decision-makers (Mono decision-maker or group decision) [28]. Despite the availability of a certain amount of information, visualization for decision-making is a real scientific and technical problem [29]. The problematic of visualization has been described by [30] and summarized in these points: ergonomics, the comprehension of basic perceptual and cognitive tasks, prior knowledge for comprehension and interpretation, education and training, scalability, measurement of intrinsic quality, aesthetics, paradigm shift from structure to dynamics, causality, visual inference and prediction, visualization of a domain of knowledge. To these problems, [31] adds the human limit of the algorithms. Scientifically, various statistical and numerical methods are used to determine the fit of mathematical models with the data used in the exploratory data analysis. Data visualization provides graphical representations for manipulating and understanding data. Data mining mechanizes the process of identifying structures useful in the data. On a technical level, the visualization is done through the so-called classic dashboards and those called smart. Smart dashboards are means of representing and consolidating information in the form of a cumulative curve, semi-logarithmic graph, sawtooth, streamer, polar 
coordinates, Gantt, etc. They are used as a medium of decision and communication [32]. The boundaries of the dashboards were analyzed in [32]. They are related to the considering of the uncertainties in the interpretation of the visualization, to the quality of the data. Several technologies are used in the literature to help implement decisions: virtual reality, augmented reality, Cave Automatic Virtual Environment (CAVE), and smart dashboards. Virtual reality is a technology that makes it possible to immerse a person in a digitally created artificial world [33]. It has the advantage of being adapted to situations where information is heterogeneous, incomplete and imprecise. In addition, the input can be conventional data, but also logical relationships or any other knowledge structures [34]. The environment can be standardized, reproducible and controllable [35]. Latency, the distortion of certain input dimensions, constitute the main limits of virtual reality [35]. Augmented reality is an interface between data and the real world. It offers interaction possibilities by combining the real world and digital elements [36]. Current limitations are identification, tracking, provision of the right information in the user's environment, real-time visualization, preparation and implementation time. From a scientific point of view, decision-making issues are the number of criteria and decision-makers as well as uncertainties about data and potential decisions. Like modeling, the visualization of decisions is the technical issue to be lifted in decision-making.

The state-of-the-art elements related to the resilience assessment reported in this paper show that no detailed study or demonstrator considered all the ambitions set by this paper. The following section describes our proposal to remove all the issues identified above.

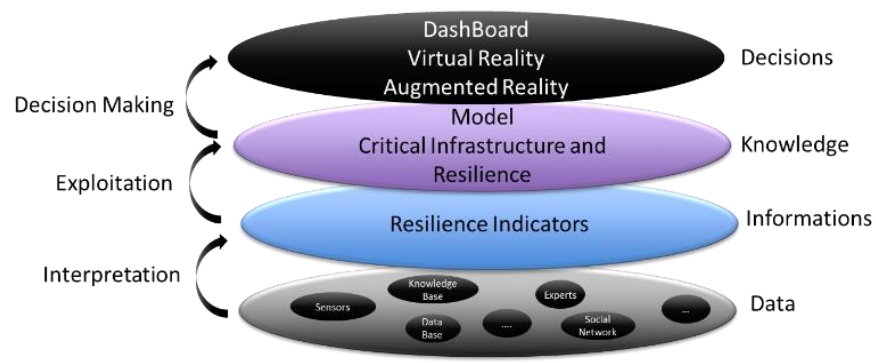

Fig 1. Proposal.

\section{Proposal}

In order to improve the current resilience of critical infrastructures, and especially to prepare the future in a context always uncertain, we propose in this paper to create and manipulate innovative representations to evaluate the resilience in continuous. The results of this paper are intended for companies 
operating critical infrastructures, communities and law enforcement, in the public and private sectors. Our proposal is a three-step process as shown in Fig 1: Data Interpretation, Indicator Exploitation, and Decision making.

\subsection{Interpretation of data}

With the concepts of smart city, industry 4.0, big data everything becomes connected to everything and vice versa. The number of information exchanged and available continues to grow. For many authors, this high level of connectivity increases the instability of the system through interdependence [37], the emergence and non-linearity of new behaviors. The consequences of sources of instability are multiplied by the cascading failures where a small disturbance can lead to large consequences. Connectivity is also characterized by the availability of a large number of data in transmission as in reception. In this paper, we propose to use Big Data from various sources to evaluate the resilience in continuous. What is then considered as the source of the problem becomes the entry point of the solution. Our proposal to be generic, will consider rules and practices specific to each domain to instrument systems by sensors, collect and store data from various sources. These rules and practices are identified in this activity through data mining techniques. This activity helps identify the relevant data sources for each selected domain. On a technical level, the data will be centralized on a platform specially created to meet the scientific objectives. For the interpretation of data, there are two trends Machine Learning using neural networks to identify indicators (subset of data) and Event-driven architecture and complex event processing [38], [39]. This last approach is used in this paper through our software suite called R-IO (https://r-iosuite.com/). In this method family, the data is considered events.

\subsection{Exploitation of indicators}

Many authors have proposed indicators for measuring resilience in the literature [6]. For the most part, the assessment of infrastructure resilience is based on a single parameter of the system [40]. Which is irrelevant from our point of view. In addition, because of the need for continuous measurement of resilience, integration of multiple dimensions, comparison to past situations, and aggregation of multiple viewpoints in evaluation, many models are unsuitable for operational use in real situations. In this activity, a resilience model is proposed and applied to the physical and behavioral model of the infrastructure. To do this, the family of simulation methods based on the resilience curve is recommended to represent the functional, behavioral and logical points of view. In this family we implement the proposition of [5]. The 
Multi Agent simulation implemented in the GAMA platform (http:/gamaplatform.org/) is used for this purpose. Several weaknesses identified in the literature are thus considered, notably: (a) the nonlinear behavior of the infrastructures, (b) the constraints and the objectives of operation, (c) the criteria (dimensions) of performance. The results of this evaluation will be an indicator of resilience for each criterion and an overall indicator of critical infrastructure. This indicator is normalized between zero (not resilient at all) and one (totally resilient).

\subsection{Decision making}

In this activity, the objective is to follow the quantitative evolution of the resilience and the evaluation of the potential actions by simulation and projection on the resilience. However, the difficulty of making a decision based on resilience before, during, or after the disruption has been highlighted by many authors [41]. Thus, to choose an action, to visualize this choice and its consequences constitute real problems. To our knowledge, there is no operational tool dedicated to this theme except the [42] approach that uses the Personal Brain tool to visualize the hierarchy of concepts related to resilience. To meet this need, it is important to propose a methodology for decision support and to design appropriate interfaces to monitor and control resilience. The entry for this activity is a continuously updated multi-criteria table. For each pair of (Decision, Criterion) the model evaluates a resilience index. The decision to recommend is the one that will be the best on all the criteria. But the reality is that no action is better on all criteria at any given time. Thus, from this table, we identify the decisions that are not dominated by any other on all the criteria (Pareto Front). This reduces all decisions into a smaller subset. The recommendation of a decision from the multicriteria table requires the integration of additional information. This information can be found in the views of different stakeholders. We then use the multicriteria method of decision PROMETHEE. The PROMETHEE method has the advantage of being able to model several points of view through preference functions. Thus, the characterization and prioritization of infrastructures is a classification problem that can be solved by using the complete classification of PROMETHEE II.

\section{$4 \quad$ Results and Discussions}

In order to illustrate the feasibility of our proposal, we constructed a use case around a fictional city. The city contains all the infrastructures that a real city in $3 \mathrm{D}$. Our job is to collect and assemble a variety of free templates to make them a coherent whole. The city is thus composed of building, road, airport, railway station, factory etc. Fig. 2 (left) gives the overview of the city under unity. From a conceptual point of view, the structure of the city is modeled in the UML diagram below shown in Fig. 2 (right). As can be seen in Fig. 2 
(right), the city is composed of networks (road, gas, electricity), Building (factory, home, public establishments, shopping center, town hall, public space) and People (Civilian, terrorist, police officer, security officer, doctors, gendarmes). Several scenarios can be simulated in this case study, but we only describe the scenario related to the risks of terrorist attack. It is possible to instantiate one or more terrorists in the city. The latter may possess three types of weapons: firearm, bomb and / or vehicle. The choice of weapons owned by a terrorist is done by the user. The damage caused will depend on the weapon used. All terrorists are represented by a class that has characteristics and behaviors that depend on the type of scenario. The behavior of the terrorist is translated into computer language in Unity. Thus, if it is equipped with a firearm, only the population category will be in danger (it is considered that bullets have no effect on other types of infrastructure). There is a delay between each shot at the population. When shooting at a target, there are two possibilities: either he misses his target, or he reaches it. The result of a shot will depend on the maximum range of the ball and its trajectory. Indeed, a target will be hit if (1) she is the first target on the trajectory of the ball; (2) the distance between it and the terrorist, at the moment of the shot, is less than or equal to the maximum range $\mathrm{P}_{\text {bal }}$ of the ball. When a target is hit, either it dies, or it is injured. So, we define two areas on the trajectory of the ball. The first zone is defined by the distance $\mathrm{P}_{1 b}<\mathrm{P}_{\text {bal }}$. If the distance between the target and the terrorist is less than or equal to $\mathrm{P} 1 \mathrm{~b}$, the target dies. The second zone is that located between $\mathrm{P}_{1 b}$ and $\mathrm{P}_{\mathrm{bal}}$. If the distance between the target and the terrorist is within this range, the target will be injured. If the target is injured, it is possible to heal it and thus avoid death.

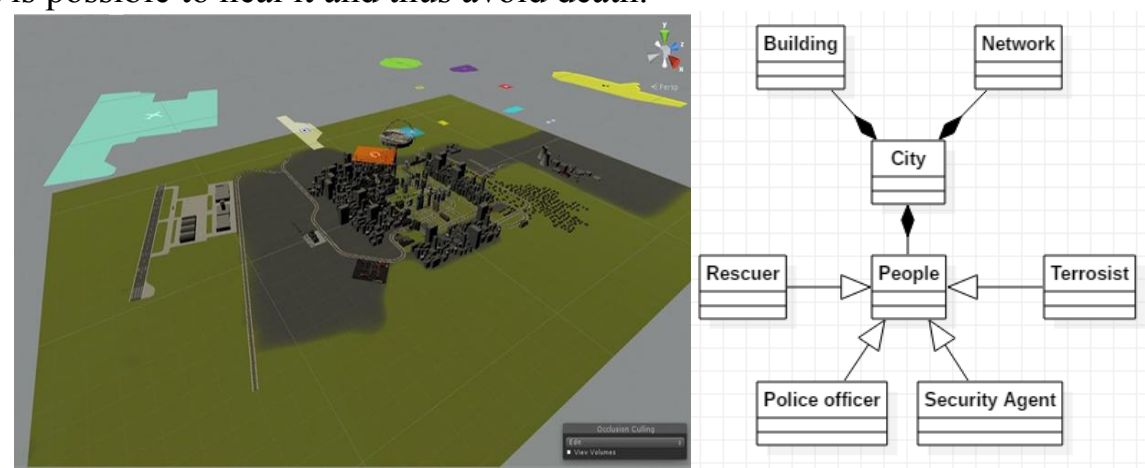

Fig 2. Case study (left) and Conceptual model (right).

\subsection{Interpretation of data}

In the case study we simulated sensors in the city. The latter make it possible to determine: (1) the number of elements damaged by type; (2) the number of destroyed elements (among the elements belonging to the city). Each of these criteria may be 
interpreted differently for each critical infrastructure. For example, for the population, the number of items destroyed corresponds to the number of dead people; while for the factories, this criterion corresponds to the number of factories destroyed. For the population, the number of damaged elements will correspond to the number of wounded; while for buildings, this criterion corresponds to the number of buildings damaged. We define the values of each criterion over time, as well as the objectives and constraints.

\subsection{Exploitation of indicators}

To exploit these indicators, we use the methodology proposed in [5]. The resilience is evaluated from several indicators called criteria. Each indicator induces a level of resilience. The value of each indicator comes from one or more sources (e.g., a sensor) and change between four parameters depending on the objectives. If the value of the indicator is between $\mathrm{f}_{\text {min }}^{\mathrm{n}}$ and $\mathrm{f}^{\mathrm{n}}{ }_{\max }$, the resilience induced by this indicator is 1 . That is to say that it has no negative influence on overall resilience. If the value of the indicator is greater than $f_{\max }$ or less than $f_{\min }$, in this case, the resilience induced by this criterion is 0 . This would mean that the negative impact of this indicator on overall resilience is maximal. If the value of the indicator is between $\mathrm{f}_{\text {min }}^{\mathrm{n}}$ and $\mathrm{f}_{\min }$ or between $\mathrm{f}_{\text {max }}^{\mathrm{n}}$ and $\mathrm{f}_{\max }$, in this case the value of the resilience induced by this criterion varies between 0 and 1 . It is calculated according to the model proposed by [5]. In this case study, we have two criteria described below: the number of damaged elements $(\mathrm{C} 1)$ and the number of destroyed elements $(\mathrm{C} 2)$. $\mathrm{C} 1$ corresponds to the total number of elements damaged in the time interval $[0, t]$. For each infrastructure in the city, the goal is to have no damaged elements. This goal does not change over time and is the same for each infrastructure. The number of damaged elements cannot be negative; so theoretically and do not exist. But we rather consider that these values will never be reached. C2 corresponds to the total number of elements destroyed in the time interval [0, t]. The objective is to have no destroyed element. This goal does not change over time and is the same for all infrastructures. The number of destroyed elements cannot be negative; so theoretically and do not exist. But we rather consider that these values will never be reached. For the population it is considered that initially, the number of elements destroyed, which in this case corresponds to the number of deaths, must not exceed 25. It is assumed that due to certain events, the city may no longer be able to withstand as much casualties. The value of the maximum stress on the number of deaths, will therefore always be between 5 and 25 deaths. For other infrastructures, it is considered that initially, the number of destroyed elements, which in this case corresponds to the number of buildings destroyed, must not exceed 3. It is assumed that due to certain events, the city may no longer be able to authorize so much material loss. 


\subsection{Decision support}

In such a scenario, the role of the user will be to limit the number of deaths by implementing several decisions: (1) Send agents to the order to neutralize the terrorist: choose the number of agents, their means of transport (service vehicle or on foot), their weapons, their destinations and their positioning in relation to the terrorist; (2) Send rescue teams to treat the wounded: to choose the composition of the teams and to assign them wounded to take charge. To neutralize a terrorist, the user must place police officers, gendarmes and / or security agents in a strategic manner. The weapons used by officers (police, gendarmes, security guards) are considered to have the same characteristics as those used by terrorists. The user will have to choose several agents and manage their movements relative to the terrorist, while because a terrorist can move and that there can be several terrorists. But also considering the time before each shot. Regarding the care of the wounded. The user must deploy rescue teams so that the wounded are treated as quickly as possible. In fact, each inhabitant has a life gauge, which will be empty if the person dies. If a person is injured, his life gauge will gradually empty and depending on the level he will indicate, he will have some time left before he dies. The user must make his choice considering the state of the various wounded and the distance between a rescue team and the various wounded. A casualty taken in charge by a rescue team can be taken to the hospital; he will be considered out of danger if he arrives at the hospital before his life gauge is emptied. The rescue teams will be able to administer care that will delay the eventual death of the injured. At each moment the overall resilience indicator is displayed on a scale of 0 to 1 . For example, Fig 3 (left) show the evolution of the criterion continuously. As expected, as soon as the value of the criterion becomes greater than the maximum limit, the resilience becomes 0 (Fig. 3 - right).

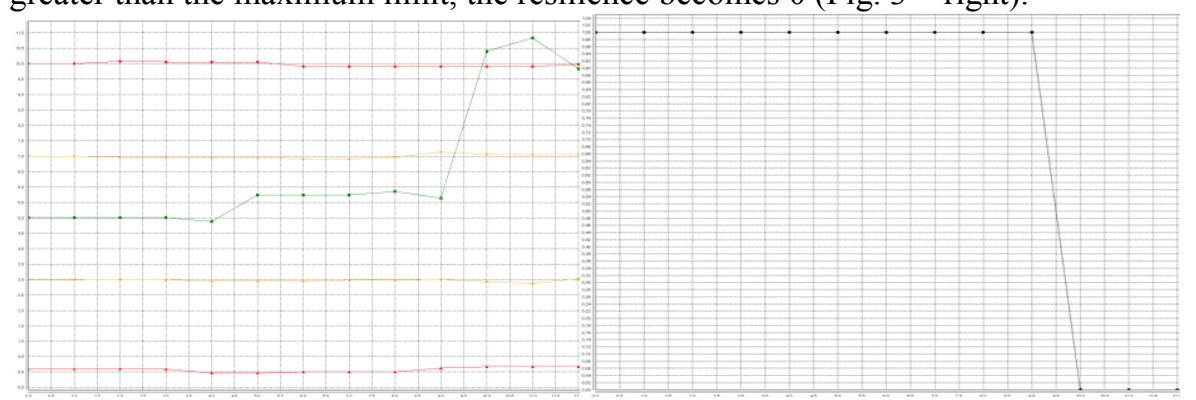

Fig 3. Example of evolution of an indicator (left) and resilience (right).

This case study has been made for the purpose of developing a virtual reality application that is only a demonstrator. It illustrates the integration virtual reality technologies into resilience management. It does not consider the actual functioning of the various infrastructures mentioned there, the aim being first to show how the decisions taken can impact the level of resilience. Numerous simplifications have been made, particularly about the development of risks, the grouping of infrastructures by category, the calculation of resilience and the choice of resilience criteria. 


\section{Conclusions}

The security and well-being of the territories depends heavily on the resilience of the hosted infrastructure. Given the growing needs of our societies, natural disasters, acts of terrorism, it is always essential to have a resilience assessment approach. The purpose of this paper was to present the approach proposed in the RESIIST project. The latter proposes a methodology and tools for (a) data collection, (b) modeling, (c) decision making (d) simulation and visualization (e) help with the implementation of decisions to evaluate in real time the resilience of critical infrastructures in order to define the possible strategies and to carry out analyzes on original criteria. Thus, with our proposal, the resilience of critical infrastructures is estimated more finely and continuously. Considering multiple dimensions, connectivity, interdependencies, integration of risk and uncertainties provides results that are very close to reality and based on a very complex reality. It greatly reduces the workload associated with low cognitive content activities in order to free up time for decision making and steering. It allows to study, evaluate and compare different technical solutions (alternatives) and to simulate different behaviors of the system. The proposal brings greater serenity to the person in charge of managing the infrastructure and reinforces the role of the human being at the center of this process. The following five contributions constitute the contributions of the methodology: (1) a generic approach, methods and tools; (2) a methodology for the identification, delimitation and prioritization of infrastructures; (3) on-going measurement indicators for resilience, based on big data; (4) a process and tool for multi-criteria decision support integrating several points of view; (5) an intelligent visualization of the system, its behavior, actions, risks in an immersive environment. Our proposal does not include some non-functional properties used in system engineering such as performance, reliability, availability etc. The integration of these properties is part of our perspective.

Acknowledgments. This paper shows a result of the RESIIST project (Résilience des infrastructures et systèmes interconnectés - Resilience of Interconnected Infrastructures and Systems https://research-gi.minesalbi.fr/display/resiist/RESIIST+Home [in French]). The RESIIST project is funded jointly by the French National Research Agency (ANR) and the General Secretary of Defense and National Security (SGDSN). The authors acknowledge these organizations for their support, and particularly, the industrial partners for the definition of the application cases.

\section{References}

1. J.-R. Ruault, "Proposition d'architecture et de processus pour la résilience des systèmes : application aux systèmes critiques à longue durée de vie," phdthesis, Université de Valenciennes et du Hainaut-Cambresis, (2015) 
2. S. Hosseini, K. Barker, and J. E. Ramirez-Marquez, "A review of definitions and measures of system resilience," Reliability Engineering \& System Safety, vol. 145, pp. 47-61, Jan. (2016).

3. J. Johanson, "Risk and vulnérability Analysis of Interdependent Technical Infrastructures," Lund University, Dept. of Measurement Technology and Industrial Electrical Engineering, (2010).

4. G. Giannopoulos, R. Filippini, and M. Schimmer, "Risk assessment methodologies for Critical Infrastructure Protection. Part I: A state of the art," JRC Technical Notes, (2012).

5. D. Kamissoko et al., "Continuous and multidimensional assessment of resilience based on functionality analysis for interconnected systems," Structure and Infrastructure Engineering, vol. 0, no. 0, pp. 1-16, Dec. (2018).

6. K. Barker et al., "Defining resilience analytics for interdependent cyber-physical-social networks," Sustainable and Resilient Infrastructure, vol. 2, no. 2, pp. 59-67, Apr. (2017).

7. E. Taysom and N. Crilly, "Resilience in Sociotechnical Systems: The Perspectives of Multiple Stakeholders," She Ji: The Journal of Design, Economics, and Innovation, vol. 3, no. 3, pp. 165-182, (2017).

8. L. F. Winfield, "The knowledge base on resilience in African-American adolescents," Pathways through adolescence: Individual development in relation to social contexts, pp. 87-118, (1995).

9. H. Stachowiak, "Allgemeine modelltheorie," (1973).

10. M. Jamshidi, "System of systems engineering - New challenges for the 21 st century," IEEE Aerospace and Electronic Systems Magazine, vol. 23, no. 5, pp. 4-19, May (2008).

11. C. Féliot, "Toward a formal theory of systems, Colloque d'Automne du LIX 2007 - CAL07 Complex Systems: Modelling, Verification and Optimization, Paris, Carré des Sciences, 3rd and 4th October 2007, (2007).

12. J. M. Penalva, La modelisation par les systemes en situation complexes. Paris, (1997).

13. P. Pederson, D. Dudenhoeffer, S. Hartley, and M. Permann, "Critical infrastructure interdependency modeling: a survey of US and international research," Idaho National Laboratory, vol. 25, p. 27, (2006).

14. INCOSE, "Survey of Model-Based Systems Engineering (MBSE) Methodologies, INCOSE TD-2007-003-01,Version/Revision: B,." (10-Jun-2008).

15. L. Lemazurier, V. Chapurlat, and A. Grossetête, "An MBSE Approach to Pass from Requirements to Functional Architecture," IFAC-PapersOnLine, vol. 50, no. 1, pp. 72607265, (2017).

16. P. Micouin, Model Based Systems Engineering: Fundamentals and Methods. John Wiley \& Sons, (2014).

17. B. Nastov, "Contribution à une méthode outillée pour la conception de langages de modélisation métier interopérables, analysables et prouvables pour l'Ingénierie Système basée sur des Modèles," (2016).

18. E. Crawley, "Introduction to System Architecture," (2007).

19. C. E. LUGLIO, "Resilience of critical infrastructures: dynamic modeling of disruptive events in a European scenario," (2017).

20. M. Ouyang, "Review on modeling and simulation of interdependent critical infrastructure systems," Reliability Engineering \& System Safety, vol. 121, pp. 43-60, Jan. (2014).

21. FMI, "Functional Mock-up Interface,". [Online]. Available: http://fmi-standard.org/. [Accessed: 26-Mar-2018], (2017).

22. HLA, "IEEE 1516-2010 - 'IEEE Standard for Modeling and Simulation (M\&S) High Level Architecture (HLA) -- Framework and Rules', IEEE Standard Association, (2010).

23. M. Desmond, "Decision criteria for the identification of alternatives in strategic environmental assessment," Impact Assessment and Project Appraisal, vol. 25, no. 4, pp. 259-269, Dec. (2007). 
24. C. J. Bacon, "The Use of Decision Criteria in Selecting Information Systems/Technology Investments," MIS Quarterly, vol. 16, no. 3, pp. 335-353, (1992).

25. H. A Simon, "Theories of Decision-Making in Economics and Behavioral Science," Am. Econ. Rev., vol. 49, pp. 253-283, Jan. (2000).

26. B. Roy, Méthodologie multicritère d'aide à la décision. Economica, (1985).

27. M. Velasquez and P. Hester, "An analysis of multi-criteria decision making methods," International Journal of Operations Research, vol. 10, pp. 56-66, May (2013).

28. E. Triantaphyllou, "Multi-Criteria Decision Making Methods," in Multi-criteria Decision Making Methods: A Comparative Study, Springer, Boston, MA, (2000), pp. 5-21.

29. B. Zhu and H. Chen, "Information Visualization for Decision Support," in Handbook on Decision Support Systems 2, Springer, Berlin, Heidelberg, (2008), pp. 699-722.

30. C. Chen, "Top 10 unsolved information visualization problems," IEEE Computer Graphics and Applications, vol. 25, no. 4, pp. 12-16, Jul. (2005).

31. 2016 at 9:35pm Posted by Larry Alton on May 8 and V. Blog, "4 Potential Problems With Data Visualization.", (2016).

32. R. Matheus, M. Janssen, and D. Maheshwari, "Data science empowering the public: Datadriven dashboards for transparent and accountable decision-making in smart cities," Government Information Quarterly, Feb. (2018).

33. J. Steuer, "Defining Virtual Reality: Dimensions Determining Telepresence," Journal of Communication, vol. 42, no. 4, pp. 73-93, Dec. (1992).

34. J. J. Valdés, "Virtual Reality Representation of Information Systems and Decision Rules: An Exploratory Technique for Understanding Data and Knowledge Structure," in Rough Sets, Fuzzy Sets, Data Mining, and Granular Computing, (2003), pp. 615-618.

35. M. Morel, B. Bideau, J. Lardy, and R. Kulpa, "Advantages and limitations of virtual reality for balance assessment and rehabilitation," Neurophysiologie Clinique/Clinical Neurophysiology, vol. 45, no. 4, pp. 315-326, Nov. (2015).

36. R. T. Azuma, "A survey of augmented reality," Presence: Teleoperators \& Virtual Environments, vol. 6, no. 4, pp. 355-385, (1997).

37. D. Kamissoko, "Decision support for infrastructure network vulnerability assessment in natural disaster crisis situations," Doctorate, University of Toulouse, University of Toulouse 1 Capitole, (2013)

38. O. Etzion and P. Niblett, Event Processing in Action, 1st ed. Greenwich, CT, USA: Manning Publications Co., (2010).

39. D. Luckham and R. Schulte, "Event Processing Glossary - Version 1.1." Jul (2008).

40. N. Yodo and P. Wang, "Engineering Resilience Quantification and System Design Implications: A Literature Survey," J. Mech. Des, vol. 138, no. 11, pp. 111408-111408-13, Sep. (2016)

41. T. McDaniels, S. Chang, D. Cole, J. Mikawoz, and H. Longstaff, "Fostering resilience to extreme events within infrastructure systems: Characterizing decision contexts for mitigation and adaptation," Global Environmental Change, vol. 18, no. 2, pp. 310-318, May (2008).

42. C. Renschler, A. E. Fraizer, L. A. Arendt, G. P. Cimellaro, A. M. Reinhorn, and M. Bruneau, "Framework for Defining and Measuring Resilience at the Community Scale: The PEOPLES Resilience Framework," Oct. (2010). 